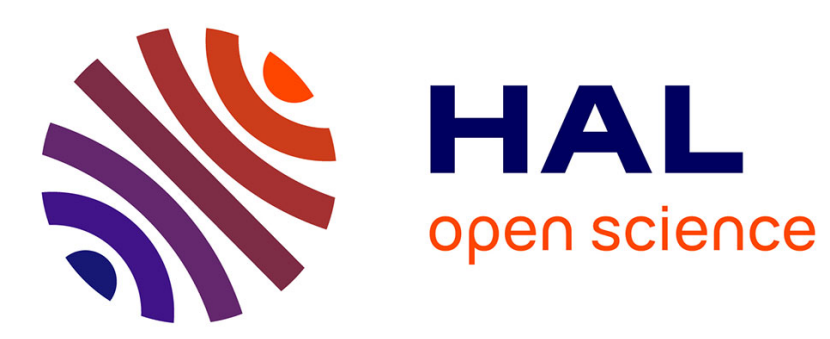

\title{
Weak Solutions within the Gradient-Incomplete Strain-Gradient Elasticity
}

\author{
V. A. Eremeyev, F. Dell'Isola
}

\section{To cite this version:}

V. A. Eremeyev, F. Dell'Isola. Weak Solutions within the Gradient-Incomplete Strain-Gradient Elasticity. Lobachevskii Journal of Mathematics, 2020, 41 (10), pp.191-197. hal-02915410

\author{
HAL Id: hal-02915410 \\ https://hal.science/hal-02915410
}

Submitted on 14 Aug 2020

HAL is a multi-disciplinary open access archive for the deposit and dissemination of scientific research documents, whether they are published or not. The documents may come from teaching and research institutions in France or abroad, or from public or private research centers.
L'archive ouverte pluridisciplinaire HAL, est destinée au dépôt et à la diffusion de documents scientifiques de niveau recherche, publiés ou non, émanant des établissements d'enseignement et de recherche français ou étrangers, des laboratoires publics ou privés. 


\title{
Weak Solutions within the Gradient-Incomplete Strain-Gradient Elasticity
}

\author{
V. A. Eremeyev ${ }^{1,2^{*}}$ and F. dell'Isola ${ }^{3,2 * *}$ \\ (Submitted by A. V. Lapin) \\ ${ }^{1}$ Faculty of Civil and Environmental Engineering, Gdańsk University of Technology, \\ Gdańsk, 80-233 Poland \\ ${ }^{2}$ Research Institute for Mechanics, National Research Lobachevsky State University of Nizhni Novgorod, \\ Nizhni Novgorod, 603950 Russia \\ ${ }^{3}$ International Research Center on Mathematics and Mechanics of Complex System (M\&MOCS), \\ Università dell'Aquila, l'Aquila, Italy
}

\begin{abstract}
In this paper we consider existence and uniqueness of the three-dimensional static boundary-value problems in the framework of so-called gradient-incomplete strain-gradient elasticity. We call the straingradient elasticity model gradient-incomplete such model where the considered strain energy density depends on displacements and only on some specific partial derivatives of displacements of first- and second-order. Such models appear as a result of homogenization of pantographic beam lattices and in some physical models. Using anisotropic Sobolev spaces we analyze the mathematical properties of weak solutions. Null-energy solutions are discussed.
\end{abstract}

Keywords and phrases: strain-gradient elasticity, weak solution, existence, uniqueness, gradient incomplete strain-gradient elasticity

\section{INTRODUCTION}

Recent progress in technologies including additive ones results in appearance of new classes of materials called metamaterials or architectured materials. Among these it is worth to mention so-called pantographic beam lattices discussed in [1,2]. Such material consists of few families of long elastic beams connected through relatively small and soft pivots. The related continual description of such materials results in a specific class of strain-gradient materials that is materials with a strain energy density dependent on first and second gradients of displacements. The general framework of the straingradient elasticity was established in the seminal papers by Toupin and Mindlin [3-5]. It is worth also to mention that an origin of such models can be found in earlier works of the continuum mechanics founders, see [6-10].

Following the Toupin-Mindlin approach a strain energy density has the form $\mathcal{W}=\mathcal{W}(\mathbf{e}, \mathbf{k})$, where $\mathbf{e}=\frac{1}{2}\left(\nabla \mathbf{u}+(\nabla \mathbf{u})^{T}\right), \mathbf{k}=\nabla \nabla \mathbf{u}$ is the strain tensor and the second gradient of $\mathbf{u}, \mathbf{u}=\left(u_{1}, u_{2}, u_{3}\right)$ is the vector of displacements, and $\nabla$ is the three-dimensional (3D) nabla-operator [11, 12].

In the framework of infinitesimal deformations $\mathcal{W}$ is a quadratic form of $\mathbf{e}$ and $\mathbf{k}$

$$
\mathcal{W}=\frac{1}{2} \mathbf{e}: \mathbf{C}: \mathbf{e}+\frac{1}{2} \mathbf{k}: \mathbf{D}: \mathbf{k},
$$

\footnotetext{
*E-mail: eremeyev .victor@gmail .com

***-mail: francescodellisola@uniroma1.it
} 
where $\mathbf{C}$ and $\mathbf{D}$ are forth- and six-order tensors of elastic moduli, respectively. Here ":" and ":" stand for scalar (inner) products in the spaces of second- and third-order tensors, respectively. The standard assumption is that $\mathbf{C}$ and $\mathbf{D}$ to be positive definite

$$
\mathbf{e}: \mathbf{C}: \mathbf{e} \geq C_{1} \mathbf{e}: \mathbf{e}, \quad \mathbf{k}: \mathbf{D}: \mathbf{k} \geq C_{2} \mathbf{k}: \mathbf{k}
$$

with $C_{1}>0, C_{2}>0$. This assumption leads to the existence and uniqueness of weak solutions of the corresponding boundary-value problems [13, 14].

The homogenization of pantographic lattices leads to a strain energy density given by (1) but without

positive definiteness. Here we have only $\mathbf{k}: \mathbf{D}: \mathbf{k} \geq 0$. As a result, the standard definition of coercitivity is violated and should be modified properly. In order to prove the existence and uniqueness of weak solutions for in-plane and out-of-plane deformations of pantographic sheets in $[15,16]$ the anisotropic Sobolev spaces were used. This class of functional spaces was introduced by Sergey Nikolskii [17], see also [18-20].

The aim of this paper is to extend the results of $[15,16]$ to three-dimensional pantographic beam lattices with perfect pivots which undergo infinitesimal deformations. The paper is organized as follows. First, we introduce a strain energy density in Section 1. In Section 2 we discuss null-energy solutions. This class of deformations includes the rigid-body deformations and should be avoided in order to get the uniqueness of solutions. Finally, in Section 3 we formulate and prove the theorems of uniqueness and existence of weak solutions. Here we use the anisotropic Sobolev spaces as an energy functional space.

\section{STRAIN ENERGY DENSITY}

Following $[15,16,21-23]$ let us briefly introduce the strain energy density of a three-dimensional pantographic lattice. It consists of three orthogonal families of elastic beams connected through small soft pivots, see Fig. 1. In what follows we neglect bending and torsional deformations of the pivots. Such pivots we call perfect ones. In addition we neglect torsional deformations of beams.

Using an approach similar to [16] we come to a strain energy density in the following form

$$
\begin{gathered}
\mathcal{W}=\frac{\mathbb{K}_{e}^{(1)}}{2} u_{1,1}^{2}+\frac{\mathbb{K}_{e}^{(2)}}{2} u_{2,2}^{2}+\frac{\mathbb{K}_{e}^{(3)}}{2} u_{3,3}^{2} \\
+\frac{\mathbb{K}_{b}^{(1)}}{2}\left(u_{2,11}^{2}+u_{3,11}^{2}\right)+\frac{\mathbb{K}_{b}^{(2)}}{2}\left(u_{1,22}^{2}+u_{3,22}^{2}\right)+\frac{\mathbb{K}_{b}^{(3)}}{2}\left(u_{1,33}^{2}+u_{2,33}^{2}\right),
\end{gathered}
$$

where the stiffness parameters $\mathbb{K}_{e}^{(i)}>0$ and $\mathbb{K}_{b}^{(i)}>0, u_{1}, u_{2}, u_{3}$ are Cartesian components of $\mathbf{u}$, $i=1,2,3$, are related to the extensional and bending stiffnesses of the beams at the interpivot scale, respectively. Hereinafter indices after comma denote derivatives with respect to $x_{i}, i=1,2,3$, where $x_{i}$ are Cartesian coordinates. So, for example, $u_{1,1}$ is the partial derivative of $u_{1}$ with respect to $x_{1}, u_{1,22}=\frac{\partial^{2} u_{1}}{\partial x_{2}^{2}}$, etc. Eq. (2) is a straightforward $3 \mathrm{D}$ generalization of $2 \mathrm{D}$ models of pantographic sheets [16].

Let us underline some similarities of (2) with other physical models known in the literature. Eq. (2) is a 3D analog of a strain energy of an extensible beams undergoing in-plane deformations

$$
\mathcal{W}=\frac{1}{2} \mathbb{K}_{e}\left(u_{1}^{\prime}\right)^{2}+\frac{1}{2} \mathbb{K}_{b}\left(u_{2}^{\prime \prime}\right)^{2},
$$

where $\mathbb{K}_{e}>0$ and $\mathbb{K}_{b}>0$ are the extensional and bending stiffnesses, $u_{1}=u_{1}(x)$ and $u_{2}=u_{2}(x)$ are longitudinal and transverse displacements, respectively, $x$ is the coordinate along the beam axis, and the prime stands for derivative with respect to $x$.

Another example is the strain energy of small deformations of the smectics A given by [24-26]

$$
\mathcal{W}=\frac{1}{2} \mathbb{B}\left(u_{, 3}\right)^{2}+\frac{1}{2} \mathbb{K}\left(u_{, 11}+u_{, 22}\right)^{2},
$$




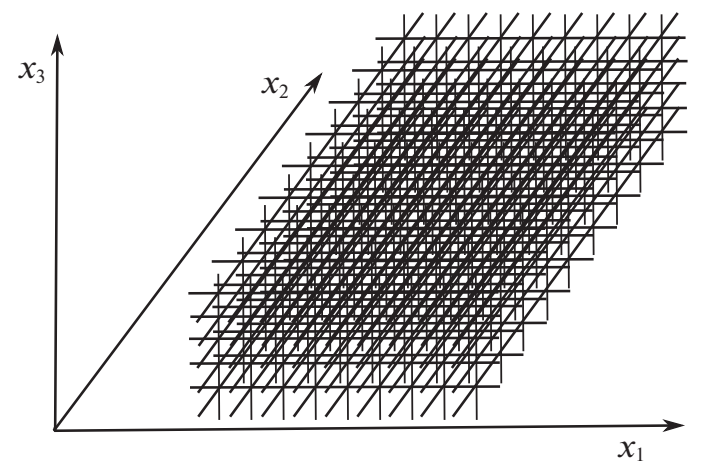

Fig. 1. Three-dimensional pantographic beam lattice.

where $\mathbb{B}$ and $\mathbb{K}$ are elastic moduli describing the longitudinal stiffness along $x_{3}$-direction and the bending stiffness of the smectic layers, respectively. Here the displacement vector has the simple form $\mathbf{u}=\left(0,0, u\left(x_{1}, x_{2}, x_{3}\right)\right.$.

Obviously, Eqs. (3) and (4) give 1D and 3D scalar examples of (2). Other physical models with nonsymmetric appearance of second-order derivatives are discussed in [27]. We call the model based on (2) gradient-incomplete as $\mathcal{W}$ does not contain all second-order derivatives. Indeed, for example, $\mathcal{W}$ contains only $u_{1,1}, u_{1,22}$, and $u_{1,22}$ while $u_{1,11}$ and mixed derivatives are absent.

\section{NULL-ENERGY SOLUTIONS}

Let us recall that for classic linear elasticity there is an energy null-space which reduces to the socalled infinitesimal rigid-body deformations. The energy null-space is a set of admissible functions for which the given strain energy density is vanishing. The analysis of these null-energy solutions is important element of the analysis of existence and uniqueness of weak solutions, see [28-30]. Let us find null-energy solutions considering solutions of

$$
\mathcal{W}=0 .
$$

In classic linear elasticity it is known that such solutions are so-called rigid-body deformations given by [30]

$$
\mathbf{u}=\mathbf{a}+\mathbf{b} \times \mathbf{x},
$$

where $\mathbf{a}$ and $\mathbf{b}$ are constant vectors, $\mathbf{x}=\left(x_{1}, x_{2}, x_{3}\right)$ is the position vector, and $\mathbf{x}$ is the cross-product. So in the linear elasticity the null-energy solutions constitute a six-dimensional subspace.

For the constitutive equation (2) we also have finite-dimensional null-space. Indeed, considering (5) we get the solutions

$$
\begin{aligned}
& u_{1}=a_{1} y z+b_{1} z+c_{1} z+d_{1}, \\
& u_{2}=a_{2} x z+b_{1} x+c_{2} z+d_{2}, \\
& u_{3}=a_{3} x y+b_{3} x+c_{3} y+d_{3},
\end{aligned}
$$

where $a_{i}, b_{i}, c_{i}$, and $d_{i}, i=1,2,3$, are constants. So for the considered model we again have finitedimensional null-energy subspace. Unlike (6) here we have 12 additional parameters so the nullspace is 12-dimensional. Obviously, (7)-(9) includes rigid-body deformations (6). Thus, the gradientincompleteness of $(2)$ results in a number of solutions with zero energy.

\section{EXISTENCE AND UNIQUENESS OF WEAK SOLUTIONS}

Let us consider an elastic solid body $B$ with the strain energy density (2). $B$ occupies a bounded volume $V$ with smooth enough boundary $S=\partial V$, see, e.g., [31-33] for the detailed requirements to $S$. Equilibrium conditions of $B$ follow from the virtual principle

$$
\delta \int_{V} \mathcal{W} d V-\delta A=0
$$


where $\delta A$ is a work of external loads, see $[8,34]$ and references therein for virtual work principle in the framework of the higher gradient elasticity models. For simplicity we consider $\delta A$ as in the case of linear elasticity [28-30]

$$
\delta A=\int_{V} \mathbf{f} \cdot \delta \mathbf{u} d V+\int_{S_{1}} \mathbf{t} \cdot \delta \mathbf{u} d S
$$

where $\mathbf{f}$ and $\mathbf{t}$ are given vector functions of body and surface forces, respectively, "." stands for scalar product of vectors, and $S_{1} \subset S$ is a part of $S$ where a traction $\mathbf{t}$ is given.

In the following we consider dimensionless form of $\mathcal{W}$ and other introduced quantities. Moreover, without loss of generality we use the following dimensionless form of $\mathcal{W}$

$$
2 \mathcal{W}=u_{1,1}^{2}+u_{2,2}^{2}+u_{3,3}^{2}+u_{2,11}^{2}+u_{3,11}^{2}+u_{1,22}^{2}+u_{3,22}^{2}+u_{1,33}^{2}+u_{2,33}^{2} .
$$

Let us consider the quadratic functional $N(\mathbf{u})$ given by $N(\mathbf{u})=\int_{V} 2 \mathcal{W} d V$. Obviously, $N(\mathbf{u})$ constitutes a seminorm, as $N(\mathbf{u})=0$ results in $(7)-(9)$.

Let us recall the definitions of norms and seminorms in the anisotropic Sobolev spaces $H_{1}=H_{2}^{(1,2,2)}$, $H_{2}=H_{2}^{(2,1,2)}$, and $H_{3}=H_{2}^{(2,2,1)}$. Here we have [17, 20]

$$
\begin{aligned}
\|u\|_{1}^{2} \equiv\|u\|_{H_{2}^{(1,2,2)}}^{2} & =\int_{V}\left(u^{2}+u_{, 1}^{2}+u_{, 22}^{2}+u_{, 33}^{2}\right) d V, \\
\|u\|_{2}^{2} \equiv\|u\|_{H_{2}^{(2,1,2)}}^{2} & =\int_{V}\left(u^{2}+u_{, 2}^{2}+u_{, 11}^{2}+u_{, 33}^{2}\right) d V, \\
\|u\|_{3}^{2} \equiv|| u \|_{H_{2}^{(2,2,1)}}^{2}= & \int_{V}\left(u^{2}+u_{, 3}^{2}+u_{, 22}^{2}+u_{, 11}^{2}\right) d V, \\
|u|_{1}^{2} \equiv|u|_{H_{2}^{(1,2,2)}}^{2} & =\int_{V}\left(u_{, 1}^{2}+u_{, 22}^{2}+u_{, 33}^{2}\right) d V, \\
|u|_{2}^{2} \equiv|u|_{H_{2}^{(2,1,2)}}^{2} & =\int_{V}\left(u_{, 2}^{2}+u_{, 11}^{2}+u_{, 33}^{2}\right) d V, \\
|u|_{3}^{2} \equiv|u|_{H_{2}^{(2,2,1)}}^{2} & =\int_{V}\left(u_{, 3}^{2}+u_{, 22}^{2}+u_{, 11}^{2}\right) d V .
\end{aligned}
$$

Obviously, with these definitions we have $N(\mathbf{u})=\left|u_{1}\right|_{1}^{2}+\left|u_{2}\right|_{2}^{2}+\left|u_{3}\right|_{3}^{2}$. In other words, $N(\mathbf{u})$ is a sum of squared seminorms in the corresponding Sobolev's spaces.

The total energy functional is given by

$$
E(\mathbf{u})=\frac{1}{2} N(\mathbf{u})-A(\mathbf{u}), \quad A(\mathbf{u})=\int_{V} \mathbf{f} \cdot \mathbf{u} d V+\int_{S_{1}} \mathbf{t} \cdot \mathbf{u} d S .
$$

In what follows we introduce a weak solution as a minimizer of $E(\mathbf{u})$ on the given energy space $H$. First, let us consider Dirichlet-type boundary conditions.

\subsection{Dirichlet-type Boundary Conditions}

Let us consider the case when $S$ is clamped

$$
\left.\mathbf{u}\right|_{S}=\mathbf{0}
$$

Boundary condition (10) prevents the appearance of null-energy solutions (7)-(9). Thus, $N(\mathbf{u})$ is a squared norm. We introduce the energy space $H_{0}$ as follows 
Definition 1. The space $H_{0}$ is the completion with respect to the norm $\|\mathbf{u}\|_{H}^{2}=N(\mathbf{u})$ of a set of vector functions $\mathbf{u}$ that are continuously differentiable on $V$ and satisfy (10). There exists the inner product in $H_{0}$

$$
\begin{gathered}
(\mathbf{u}, \mathbf{v})_{H}=\int_{V}\left(u_{1,1} v_{1,1}+u_{2,2} v_{2,2}+u_{3,3} v_{3,3}+u_{2,11} v_{2,11}+u_{3,11} v_{3,11}+u_{1,22} v_{1,22}\right. \\
\left.+u_{3,22} v_{3,22}+u_{1,33} v_{1,33}+u_{2,33} v_{2,33}\right) d V .
\end{gathered}
$$

So $H_{0}$ is a Hilbert space. Moreover, here

$$
H_{0}=\stackrel{\circ}{H}_{2}^{(1,2,2)}(V) \oplus \stackrel{\circ}{H}{ }_{2}^{(2,1,2)}(V) \oplus \stackrel{\circ}{H}_{2}^{(2,2,1)}(V),
$$

where $\stackrel{\circ}{H}_{2}^{\ell}(V)$ is the completion with the norm $\|\cdot\|_{H_{2}^{\ell}(V)}$ od a set of functions that satisfy (10), and $\ell$ is a multi-index, $\ell=(1,2,2),(2,1,2),(2,2,1)$.

Now we introduce a weak (generalized) solution.

Definition 2. The element $\mathbf{u} \in H_{0}$ is a weak of the equilibrium problem for the body $B$ with external loads $\mathbf{f}$ if it minimizes $E(\mathbf{u})$ on the energy space $H_{0}$.

Calculating the first variation of $E(\mathbf{u})$ we conclude that a weak solution satisfies the equation

$$
(\mathbf{u}, \mathbf{v})_{H}-\int_{V} \mathbf{f} \cdot \mathbf{v} d V=0, \quad \forall \mathbf{v} \in H_{0} .
$$

For simplicity we assume that $\mathbf{f} \in L_{2}(V)$. Then we have

Theorem 1. A weak solution $\mathbf{u} \in H_{0}$ to equation (12) exists and unique.

Proof. The proof mimics one given in $[15,16]$ and we omit it for brevity.

\subsection{Mixed Boundary Conditions}

Let us now relax the kinematic constraints assuming that only a part $S_{2} \subset S$ is clamped while on the rest $S_{1}=S \backslash S_{2}$ a traction $\mathbf{t}$ is given:

$$
\left.\mathbf{u}\right|_{S_{2}}=\mathbf{0}
$$

For simplicity we assume that $\mathbf{t} \in L_{2}\left(S_{2}\right)$.

Unlike linear elasticity where (13) prevents rigid-body deformations, this is not the case in the framework of the gradient-incomplete strain-gradient elasticity, see few examples [16]. Following [15, 16] we assume that assumed boundary conditions are non-singular. In other words we apply such kinematical constraints (13) that are sufficient for absence of null-energy deformations. In this case we define again a weak solution $\mathbf{u} \in H$ as a minimizer of the total energy functional $E(\mathbf{u})$. Here the energy space $H$ the completion of a set of differentiable functions, with respect to the norm $\|\cdot\|_{H}$, that satisfy (13). $H$ is a Hilbert space with inner product (11). Moreover, $H=H_{2}^{(1,2,2)}(V) \oplus H_{2}^{(2,1,2)}(V) \oplus$ $H_{2}^{(2,2,1)}(V)$. Similarly to [16, 30] we can prove

Theorem 2. Suppose the boundary conditions are non-singular. There exists a weak solution $\mathbf{u} \in H$ to the equilibrium problem that minimizes the total energy functional $E(\mathbf{u})$ in $H$ and satisfies the integral equation

$$
(\mathbf{u}, \mathbf{v})_{H}-\int_{V} \mathbf{f} \cdot \mathbf{v} d V-\int_{S_{1}} \mathbf{t} \cdot \mathbf{v} d S=0, \quad \forall \mathbf{v} \in H .
$$

The weak solution is unique. 


\section{CONCLUSIONS}

Following $[15,16]$ we discussed the existence and uniqueness of weak solutions in the framework of the spatial gradient-incomplete strain-gradient elasticity. To this end we used the anisotropic Sobolev spaces. In this paper we considered non-singular boundary conditions that prevent null-energy solutions. The case of singular boundary conditions and free bodies can be also studied as in [30]. Clearly, in this case a weak solution is unique up to arbitrary rigid-body deformations given by (7)-(9) whereas external loadings including hyper stresses should be orthogonal to these deformations.

\section{FUNDING}

Authors acknowledge the support of the Government of the Russian Federation (contract no. 14.Y26.31.0031).

\section{REFERENCES}

1. F. dell'Isola, P. Seppecher, J. J. Alibert, T. Lekszycki, R. Grygoruk, M. Pawlikowski, D. Steigmann, I. Giorgio, U. Andreaus, E. Turco, M. Gołaszewski, N. Rizzi, C. Boutin, V. A. Eremeyev, A. Misra, et al., "Pantographic metamaterials: An example of mathematically driven design and of its technological challenges," Continuum Mech. Thermodyn. 31, 851-884 (2019).

2. F. dell'Isola, P. Seppecher, M. Spagnuolo, E. Barchiesi, F. Hild, T. Lekszycki, I. Giorgio, L. Placidi, U. Andreaus, M. Cuomo, S. R. Eugster, A. Pfaff, K. Hoschke, R. Langkemper, E. Turco, et al., "Advances in pantographic structures: Design, manufacturing, models, experiments and image analyses," Continuum Mech. Thermodyn. 31, 1231-1282 (2019).

3. R. D. Mindlin, "Micro-structure in linear elasticity," Arch. Ration. Mech. Anal. 16, 51-78 (1964).

4. R. D. Mindlin and N. N. Eshel, "On first strain-gradient theories in linear elasticity," Int. J. Solids Struct. 4, 109-124 (1968).

5. R. A. Toupin, "Elastic materials with couple-stresses," Arch. Ration. Mech. Anal. 11, 385-414 (1962).

6. G. A. Maugin, "Generalized continuum mechanics: Various paths," in Continuum Mechanics Through the Twentieth Century: A Concise Historical (Perspective Springer, Dordrecht, 2013), pp. 223-241.

7. G. A. Maugin, Non-Classical Continuum Mechanics: A Dictionary (Springer, Singapore, 2017).

8. N. Auffray, F. dell'Isola, V. A. Eremeyev, A. Madeo, and G. Rosi, "Analytical continuum mechanics à la Hamilton-Piola least action principle for second gradient continua and capillary fluids," Math. Mech. Solids 20, 375-417 (2015).

9. F. dell'Isola, U. Andreaus, and L. Placidi, "At the origins and in the vanguard of peridynamics, non-local and higher-gradient continuum mechanics: An underestimated and still topical contribution of Gabrio Piola," Math. Mech. Solids 20, 887-928 (2015).

10. F. dell'Isola, A. Della Corte, and I. Giorgio, "Higher-gradient continua: The legacy of Piola, Mindlin, Sedov and Toupin and some future research perspectives," Math. Mech. Solids 22, 852-872 (2016).

11. J. G. Simmonds, A Brief on Tensor Analysis, 2nd ed. (Springer, New Yourk, 1994).

12. V. A. Eremeyev, M. J. Cloud, and L. P. Lebedev, Applications of Tensor Analysis in Continuum Mechanics (World Scientific, New Jersey, 2018).

13. T. J. Healey and S. Krömer, "Injective weak solutions in second-gradient nonlinear elasticity," ESAIM: Control, Optim. Calculus Variat. 15, 863-871 (2009).

14. A. Mareno and T. J. Healey, "Global continuation in second-gradient nonlinear elasticity," SIAM J. Math. Anal. 38, 103-115 (2006).

15. V. A. Eremeyev, F. dell'Isola, C. Boutin, and D. Steigmann, "Linear pantographic sheets: Existence and uniqueness of weak solutions," J. Elasticity 132, 175-196 (2018).

16. V. A. Eremeyev, F. S. Alzahrani, A. Cazzani, F. dell'Isola, T. Hayat, E. Turco, and V. KonopińskaZmysłowska, "On existence and uniqueness of weak solutions for linear pantographic beam lattices models," Continuum Mech. Thermodyn. 31, 1843-1861 (2019).

17. S. M. Nikol'skii, "On imbedding, continuation and approximation theorems for differentiable functions of several variables," Russ. Math. Surv. 16 (5), 55-104 (1961).

18. O. V. Besov, V. P. II'in, and S. M. Nikol'skii, Integral Representations of Functions and Imbedding Theorems (Wiley, New York, 1978), Vol. 1.

19. O. V. Besov, V. P. II'in, and S. M. Nikol'skii, Integral Representations of Functions and Imbedding Theorems (Wiley, New York, 1979), Vol. 2.

20. H. Triebel, Theory of Function Spaces III, Vol. 100 of Monographs in Mathematics (Birkhäuser, Basel, 2006). 
21. C. Boutin, F. dell'Isola, I. Giorgio, and L. Placidi, "Linear pantographic sheets: Asymptotic micro-macro models identification," Math. Mech. Complex Syst. 5, 127-162 (2017).

22. L. Placidi, U. Andreaus, A. Della Corte, and T. Lekszycki, "Gedanken experiments for the determination of two-dimensional linear second gradient elasticity coefficients," Zeitschr. Angew. Math. Phys. 66, 3699-3725 (2015).

23. L. Placidi, L. Greco, S. Bucci, E. Turco, N. L. Rizzi, "A second gradient formulation for a 2D fabric sheet with inextensible fibres," Zeitschr. Angew. Math. Phys. 67 (5), 114 (2016).

24. S. Chandrasekhar, Liquid Crystals (Cambridge Univ. Press, Cambridge, 1977).

25. P. G. de Gennes and J. Prost, The Physics of Liquid Crystals, 2nd ed. (Clarendon, Oxford, 1993).

26. P. Oswald and P. Pieranski, Smectic and Columnar Liquid Crystals, The Liquid Crystals Book Series (CRC, Boca Raton, 2006).

27. V. A. Eremeyev and F. dell'Isola, "A note on reduced strain gradient elasticity," in Generalized Models and Non-classical Approaches in Complex Materials 1, Ed. by H. Altenbach, J. Pouget, M. Rousseau, B. Collet, and T. Michelitsch, Vol. 89 of Advanced Structured Materials (Springer, Cham, 2018), pp. 301310.

28. G. Fichera, "Existence theorems in elasticity," in Handbuch der Physik, Ed. by S. Flügge (Springer, Berlin, 1972), Vol. VIa/2, pp. 347-389.

29. P. G. Ciarlet, Mathematical Elasticity, Three-Dimensional Elasticity (North-Holland, Amsterdam, 1988), Vol. 1.

30. V. A. Eremeyev and L. P. Lebedev, "Existence of weak solutions in elasticity," Math. Mech. Solids 18, 204217 (2013).

31. R. A. Adams and J. J. F. Fournier, Sobolev Spaces, 2nd ed. (Academic, Amsterdam, 2003).

32. L. P. Lebedev and I. I. Vorovich, Functional Analysis in Mechanics (Springer, New York, 2003).

33. V. Maz'ya, Sobolev Spaces: With Applications to Elliptic Partial Differential Equations, Vol. 342 of Grundlehren der mathematischen Wissenschaften, 2nd ed. (Springer, Berlin, 2011).

34. B. E Abali, W. H. Müller, and F. dell'Isola, "Theory and computation of higher gradient elasticity theories based on action principles,” Arch. Appl. Mech. 87, 1495-1510 (2017). 